(C2005 IEEE. Personal use of this material is permitted. However, permission to reprint/republish this material for advertising or promotional purposes or for creating new collective works for resale or redistribution to servers or lists, or to reuse any copyrighted component of this work in other works must be obtained from the IEEE. 


\title{
Ontology-based Support for Human Disease Study
}

\author{
Maja Hadzic, Elizabeth Chang \\ Curtin University of Technology \\ School of Information Systems \\ GPO Box U1987, Perth WA 6845 \\ Australia \\ E-mail:maja.hadzic@,cbs.curtin.edu.au, elizabeth.chang@.cbs.curtin.edu.au
}

\begin{abstract}
In this paper, we discuss an ontology-based system and approach that provides interoperability support for research in, and diagnosis of, human disease. The proposed solution incorporates a prototype for a Generic Human Disease Ontology that contains common general information regarding human diseases. This proposed Generic Human Disease Ontology represents the information in 4 'dimensions': (1) disease types, (2) phenotype (observable characteristics of an organism) or symptoms (3) causes related to the disease, namely genetic causes (genes, gene complexes and DNA regions of interest) and environmental causes (such as stress, family condition, drugs, climate etc.), and (4) treatments available for the disease such as drug therapy, chemotherapy, physiotherapy, psychotherapy, surgery etc. The Generic Human Disease Ontology aims to support also the study of complex disorders caused by many different factors simultaneously e.g. the case of manic depressive disorders. We illustrate how this Generic Human Disease Ontology helps to produce Specific Human Disease Ontologies on request, as support tool for physicians and medical researchers.
\end{abstract}

\section{Introduction}

A body of formally represented knowledge is based on conceptualization. Conceptualization is an abstract, simplified view of the world that we wish to represent for some purpose, usually involving computers. It consists of a set of objects, concepts and other entities about which knowledge is being expressed (often called the universe of discourse) and of relationships that hold among them. Every formal knowledge model is committed to some conceptualization, implicitly or explicitly. An explicit specification of this agreed conceptualization is called ontology [9].

Computer based ontologies, as shared formal conceptualizations of domain knowledge, constitute an essential resource for enabling interoperation in an open environment such as the Internet. In this paper, we illustrate how ontologies can be developed for the knowledge domain of biomedical and bioengineering research. It is a post internet domain which enjoys an unusually large number of high-quality, complex, but extremely heterogeneous information resources, which furthermore are often made available through site-specific services only. We chose the application domain of human disease research and control since it necessarily involves resources of medical, genetic, environmental and treatment data. A characteristic of the domain is that trusted databases exist but their schemas are often poorly or not documented for outsiders, and explicit agreement about their contents is therefore rare.

Worldwide many research groups are studying the relationship of genes and genome to diagnostics of disease or disorders. They may use laboratory based experimental research combined with computer aided, semi automated, bioinformation research. Recently automated techniques like bioontologies started playing an important role in bioinformatics research as researchers increasingly wish to combine different techniques to perform the disease study. Typically, a research team examines one factor for one type of disorder at a time. But in case of complex disorders, one needs to look at most, if not all, different factors simultaneously. We need a tool that combines and examines all genetic factors together, all environmental factors together and all genetic and environmental factors together.

Traditionally, the knowledge base in biomedicine has resided within the heads of experienced biochemists and doctors, scientists who have devoted much study to becoming experts in their particular domain of study. This approach worked well in the past when considerable effort was needed to tease new data out of biochemical experiments and the flow of data was not so great as to overwhelm the expert. However, this situation is rapidly changing. New modern experimental techniques are providing huge, rapidly accumulating amounts of information. This information is stored and analyzed in different biomedical databases. The biomedical community is a distributed one and a large body of knowledge has become available through the Internet. The information sources have complete autonomy and they are continually extending their content. Also, each area of biomedical research generates its own database. In 
this community, sharing of information inside an area as well as between different research areas is essential and data from one source often must be combined with data from other sources to give users the information they desire. This network of biomedical databases forms a loose federation of autonomous, distributed, heterogeneous data repositories ripe for information integration.

Accurate predictions can only be made against a knowledge base, and the bigger the knowledge base the more accurate the predictions that can be made. However, the size of the existing knowledge base is too large for any human to assimilate. Therefore, predictions are only being made against a small subset of the available knowledge and information is being neglected. There is clearly a need to create systems that can apply the knowledge in the head of a domain expert to biological data.

Classical techniques and methodologies are largely inadequate because of the inherent autonomous and heterogeneous nature of the information resources which force applications to share data and respective services, often without prior knowledge of their structures functionality.

Developing ontology, as a unifying resource, would be of great importance for the researcher to be able to solve this problem. Only then, researchers will be able to look at all different factors causing human disorders simultaneously and identify possible links. Now, this research is limited by the number of factors one can examine [17].

In Section 2 (Baseline and Related Work), we discuss semi-automated and automated techniques used in study of human diseases. Section 3 (Principles of building a Generic Human Disease Ontology) describes the four main branches of Generic Human Disease Ontology made by using DOGMA Modeler. Section 4 (Illustration of use and application of Generic Human Disease Ontology to derive Specific Human Disease Ontologies) illustrates on the examples the ontology as a tool for physicians (section 4.1) and for researchers (section 4.2). Section 5 (Conclusions and further work), gives final remarks and discusses future plans.

\section{Baseline and Related Work}

The most commonly used semi-automated techniques are Linkage Analysis and DNA Sequencing [1]. Predictive Methods, predictive methods such as finding a gene within a DNA sequence or predicting properties of a protein from its sequence can be performed once DNA and/or protein sequence is known [3].

Unified Medical Language System (UMLS) project develops and distributes multi-purpose, electronic 'Knowledge Sources' and associated lexical programs.
System developers can use the UMLS products to enhance their applications in systems focused on patient data, digital libraries, Web and bibliographic retrieval, natural language processing, and decision support. Researchers will find the UMLS products useful in investigating knowledge representation and retrieval questions [5].

Recently, emerging research has moved to Ontology based bioinformatics research. Biochemistry is rich in taxonomies, such as the enzyme classification and species taxonomies. Being taxonomies, they only use a hierarchy. The ontologies tend to be richer in their use of relationships hence their inclusion. Ontology based work includes: the Riboweb ontology, the Gene Ontology (GO), the TAMBIS Ontology (TaO) and L\&C's LinkBase ${ }^{\circledR}$.

The Riboweb Ontology [2] is a resource whose primary aim is to facilitate the construction of threedimensional models of ribosomal components and compare the results with existing studies. This ontology is helping solve the problem of the structure and representation of a cellular component, ribosome.

The Gene Ontology (GO) [8] was initially created to reflect fruit fly Drosophila gene function via the Flybase Database, but has expanded to encompass mouse, yeast and gene expression databases. GO seeks to capture information about the role of gene products within an organism. This ontology does not explain how all identified genes are related to human disorders.

The TAMBIS Ontology ( $\mathrm{TaO})$, Transparent Access to Multiple Bioinformatics Information Sources [16], uses ontology to enable biologists to ask questions over multiple external databases using a common query interface. TAMBIS Ontology gives information regarding different proteins and nucleic acids but it does not relate them to human disease study.

In summary, the Riboweb Ontology can be helpful for scientists studying ribosome related diseases, but it doesn't support study of other much more numerous diseases. Gene Ontology provides us with information about all genes within an organism and the TAMBIS Ontology represents all nucleic acids and proteins, but scientists studying a particular disease are only interested in genes and proteins responsible for that particular disease.

LinKBase $^{\circledR}$ by $L \& C$ represents a case apart, and incorporates recent results involving a very large commercially available formal domain ontology. It is reported [14] to currently contain over 5.000 .000 knowledge entities of various types: concepts, relationships, terms etc. These entities represent medicine in a way that can be understood by algorithms. Consistency is maintained through a description-logic based knowledge system called LinKFactory ${ }^{\circledR}$. This system is a framework consisting of a number of components that can be freely linked together in a 
dynamic workspace. The components either give a view on the ontology or allow manipulation of it. Examples of its software components are a concept tree, a concept criteria editor, a definition editor, a concept terms editor, a concept relation finder etc. The ontology is still growing.

The above mentioned ontologies are all representing different knowledge domains, but just a minor portion of their results is presently directly related to our work. As these ontologies, their concepts and relationships, are not specifically geared to be used in the case of human disorders, and would require considerable realignment for this purpose. Some research has started supporting disease analysis [7], however, but as yet they only provide a scientist with a simple hierarchical and manually written vocabulary. However, we shall be investigating in the near future the possibilities of formal alignment of our prototype Generic Human Disease Ontology with some of the above, notably LinkBase.

\section{Principles of building a Generic Human Disease Ontology}

In the sequel we shall adopt the DOGMA formalism [13], [15] for the description and terminology involving ontologies, as its semantics are based on agreements in natural language, and represents the real world merely as a large set of lexons, elementary plausible facts that may be true in a given context.

Ontological commitments are formal agreements (expressed in DOGMA as views, rules, and constraints) to use the shared vocabulary in a coherent and consistent manner. Shared vocabulary is different for different knowledge domains. Our knowledge domain is going to have its own vocabulary written in an ontological lexicon. An ontology base consists of lexons, expressing (usually linguistically derived) facts between terms. Terms are often organized hierarchically in taxonomy, by promoting the subsumption fact into an implicit, special, and axiomatically defined relationship. Facts in DOGMA are always true only within a context, defined for any lexon as carried by an identifiable source, usually a document.

The constructed Generic Human Disease Ontology has four main branches: (1) types, describing different types of a disorder; (2) phenotype, describing symptoms of a disease; (3) causes responsible for that disorder which can be environmental and/or genetic; (4) treatments, giving an overview of all treatments possible for that particular disease as well as treatment efficiency. This ontology helps to generate Specific Human Disease Ontologies on request as illustrated in section 4. In the Figure 1, we illustrate four main branches of the Generic Human Disease Ontology. The Generic Human Disease Ontology has been build using DOGMA Modeler tool. Terms within Generic Human Disease Ontology are much more numerous than shown and are validated for existence against concepts from a biomedical lexicon such as UMLS Metathesaurus [5].

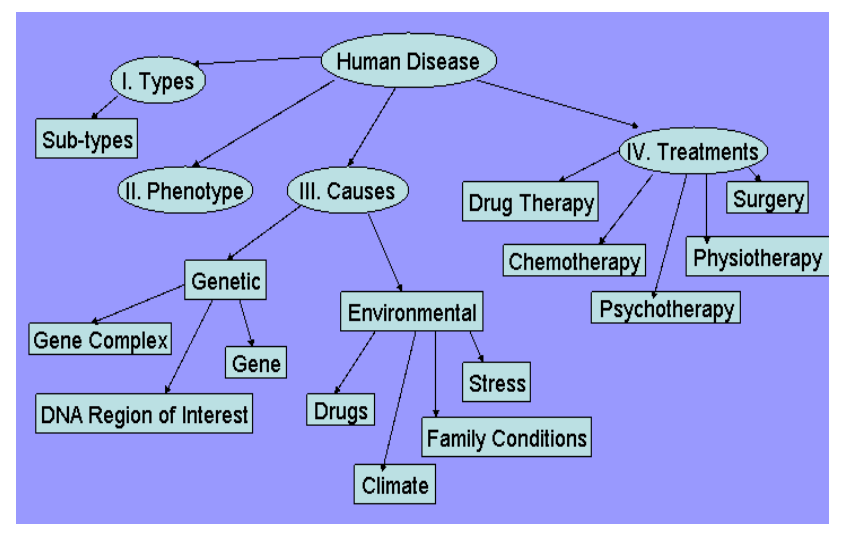

Figure 1: Generic Human Disease Ontology and its four main subontologies: types, phenotype (symptoms), causes and treatments.

As a simple example, we develop a small generic ontology representing the main concepts, identified in a given (implicit) context. Consider a vocabulary $\mathbf{V}=\mathbf{( T}$, $\mathbf{R})$ where $\mathbf{T}$ is a set of terms denoting concepts, and $\mathbf{R}$ is a set of relationship names. Let $\mathbf{T}=$ human disease, type, subtype, phenotype, treatment, drug therapy, chemotherapy, physiotherapy, surgery, psychotherapy, cause, genetic, gene, gene complex, DNA region of interest, environment, stress, climate, family conditions, drugs \} that represent the lexicon of user's world of diseases, and $\mathbf{R}=$ has, is of, is a, is caused by, is responsible for, is cured by, cures, shows, characterizes\} that represent relationships (roles) for this domain.

The ontology explains that a disease may have (1) different types which also may be further divided into subtypes. Each disease is caused by (3) cause/s which can be genetic (genotype) or environmental. Genetic causes can be a mutated gene, a complex of genes or DNA region of interest (a region in the DNA sequence that potentially contains a gene responsible for the disease and needs to be further examined). Environmental causes can be stress, climate, drugs or family conditions. For each disease, there is (2) a corresponding phenotype of observable characteristics of an ill individual; and (4) treatments possible for the disorder that can be drug therapy, chemotherapy, surgery, psychotherapy or physiotherapy.

Unlike traditional Artificial Intelligence ontology engineering proposals, which consider ontology as being a single unit holding both conceptual related objects and rules together, the approach to ontology engineering adopted by DOGMA is based on the principle of a double 
articulation. An ontology is decomposed into an ontology base, which holds intuitive conceptualizations of a domain, and a layer of ontological commitments, where each commitment holds a set of domain rules.

First, we objectify all possible concepts implied by all data resources. Secondly, we populate the ontology base by constructing lexons from these concepts reflecting all valid statements within the resources. Next, we construct commitments, each applying rules and constraints to a selection of lexons. For each participating data resource, we create a commitment that captures and formalizes all the knowledge statements of this resource.

The ontology base holds binary conceptual relations, called lexons, which are intuitive statements or facts within a specific real world domain. A lexon is formally described as $<\gamma$, term 1 , role, co - role, term $2>$, where $\gamma$ is an abstract context identifier used to group lexons that are intuitively related to each other within a conceptualization of a domain. We take following examples:

- $\quad<$ human diseases, disease, has, is of, type >

This means that in the context of human diseases 'disease has type' and 'type is of disease'.

- <human diseases, disease, shows, characterizes, phenotype $>$

This means that in the context of human diseases 'disease shows phenotype' and 'phenotype characterizes disease'.

- <human diseases, disease, is caused by, is responsible for, cause $>$

This means that in the context of human diseases 'disease is caused by cause' and 'cause is responsible for disease'.

- <human diseases, disease, is cured by, cures, treatment>

This means that 'disease is cured by treatment' and 'treatment cures disease'.

The commitment layer contains a set of interpretations, also known as commitments. Each commitment provides a specific interpretation to a subset of lexons in the ontology base by means of rules and constraints. Currently, the rules of a commitment in DOGMA are described by the ORM Markup Language (ORMML), which supports the rules and constraints of the ORMnotation, which is the conceptual modeling language proposed by Halpin [10]. As examples we take the following commitments:

- 'each disease has zero-or-more type'

- 'each disease shows at least one phenotype'

- 'each disease is caused by at least one cause'

- 'each disease is cured by zero-or-more treatments'

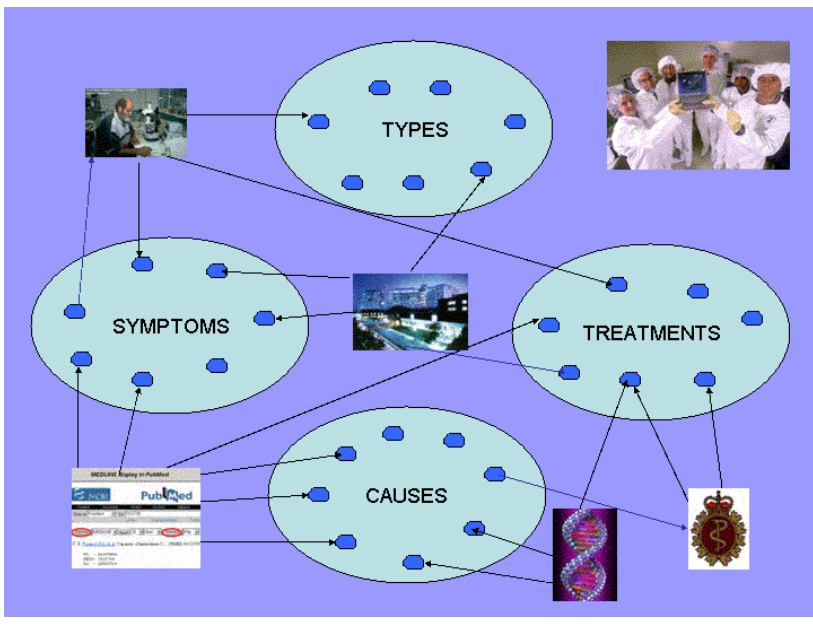

Figure 2: Combining of the information from different databases worldwide into the four dimensions of Generic Disease Ontology

The conceptual framework of our Generic Human Disease Ontology methodology and prototype will be based on such a formal theory of ontology. Indeed, we will extract relevant information from publication and medical databases, DNA and protein databases, research institutes, health departments, hospitals etc (Figure 2). Upon the analysis and combination of the information, the result will be presented in a way that makes it easier for the user to have an overview of the up-to-date knowledge about a specific disorder. Use of ontologies provides us with a more controlled and systematic way to perform information retrieval. Moreover, the inherited organization of ontologies adds taxonomical context to search results, making it easier for the researcher to spot conceptual relationships in data. The latter fact is important for instance in the case of complex human disorders where one looks for relationships between different factors that are simultaneously responsible for each of the many types of disorders.

The four 'dimensions' (phenotype, cause, treatment and type) are built for a different purpose and are orthogonal to each other. The 'Types' sub-ontology is more a classifying ontology and is strongly hierarchically supported. It does not provide a user with much scientific information. This ontology is based on classification. The 'Phenotype' sub-ontology is more descriptive than the others and is based on observation and diagnosing characteristics of ill individual. The 'Cause' sub-ontology is providing a user with scientifically proven facts and is strongly based on scientific research. The 'Treatment' sub-ontology is a combination of classifying and research ontology. Modeling available treatments is research work 
but, for example all the discovered drugs can be further hierarchically classified. All four 'dimensions' are different from each other and each 'dimension' is unique. But jointly they give an overall picture and a good overview of knowledge on a human disorder.

\section{Illustration of use and application of Generic Human Disease Ontology to derive Specific Human Disease Ontologies}

We present three examples for the purpose of illustration of use and application of Generic Human Disease Ontology to generate on request Specific Human Disease Ontologies. The Generic Human Disease Ontology, which serves as a template, has four main branches: types, causes, symptoms and treatments. Computer services then feed applications committed to this ontology with relevant data required by a user.

The examples are intended to show typical, common problems researchers and physicians encounter. Researchers are constantly searching for and adding more information to the already existing pool of knowledge regarding a particular disorder. Physicians are directly in contact with patients and are using all significant information to help and treat the patients. Researchers and physicians are strongly connected because they are working towards the same goal, but on different knowledge levels.

\subsection{Ontology as support tool for physicians}

If a medical professional queries the system, she/he will mainly be interested in two of the four components of our system, namely symptoms and possible treatments of a particular disorder. There are some exceptions to this rule, such as in the next use case.

Use case one: Physician cannot identify the disease. A physician may have a patient showing some symptoms of a disease but he may not be able to say what kind of disease it is. At this stage, it is recommended to keep three components involved in the search (symptoms (phenotype), causes and treatments). In this case, the derived Specific Human Disease Ontologies have the 'phenotype', 'causes' and 'treatments' branches.

By entering the symptoms into the system, she/he may be able to retrieve the information regarding that disease. It is also possible that different diseases are showing the same or similar symptoms, so that the physician retrieves more than one Specific Human Disease Ontology (in Figure 3 we show two different Specific Human Disease Ontologies). In that case, it may become useful to look for some significance in the causes of the disorders.

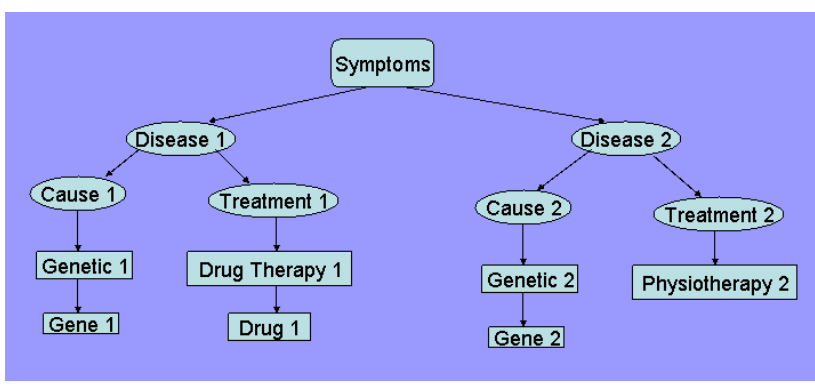

Figure 3: Two different diseases showing same symptoms caused by mutations of different genes and differently treated

For example, in case of disease_1, gene_1 is mutated and thus causes this disorder. And disease_ 2 is caused by mutation of gene 2 . The physician can do the screening of the patients DNA to check if gene_1 or gene_2 is mutated. If mutation found in gene 1, the patient has disease_1 and can be treated by drug 1 . Otherwise, if gene_2 is mutated the patient suffers from disease_2 and physiotherapy only is enough for the treatment of this patient. Only when the patient is correctly diagnosed, the physician may consider possible treatments for the patient. Our information system therefore also reduces the risk of misdiagnosis.

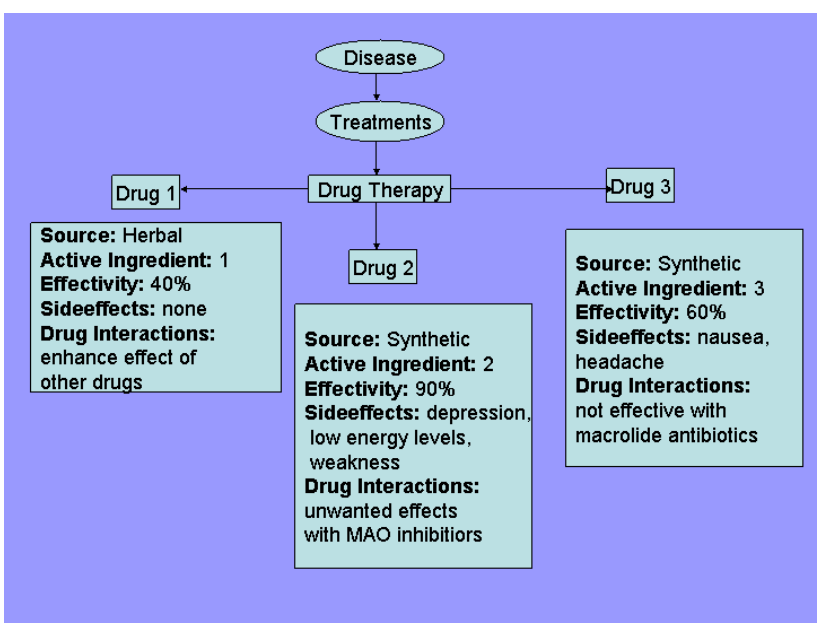

Figure 4: Different drugs target same disease

Use case two: Physician can identify the disease and wants to consider possible treatments. It is common that there is more than one (drug) treatment possible for a particular disease (see Figure 4). A physician will wish to look at all the options possible before choosing one. Choosing medication is subjective because not all the people respond in the same way to the same medication. At this point a medical professional might consult our ontology-based information system to do a onecomponent search (treatments). In this case, the derived 
Specific Human Disease Ontology has only the 'treatment' branch.

We represent such a Specific Human Disease Ontology presenting three different drugs with different properties (source, active ingredient, affectivity, side effects, drug interactions etc.). Those different drugs are used to treat the same disease.

\subsection{Ontologies as Tools for Researchers}

When a biomedical researcher uses our system, she/he will in general mainly be interested in one specific of the four possible components of our system, namely causes or treatments depending on her/his research area. A Researcher working on drug discovery would be more interested in the 'treatment' branch. We show another example where the derived Specific Human Disease Ontology has only the 'cause' branch.

Use case three: Researcher examines possible causes of a disorder. Often not all the causes responsible for a particular disorder are known, e.g. in the case of manicdepression.

By querying our system and getting back significant information systematically represented (see Figure 5), the researcher is able to identify some regions of interest in the DNA sequence such as regions 2p13-16, 10q21-24, 12q23-24, 17q11-12 and Xq24-26 on chromosomes 2, 10, 12,17 and $\mathrm{X}$ respectively [4], [6], [11], [12]. Those regions need to be further examined in order to find a gene and a mutation inside that gene.

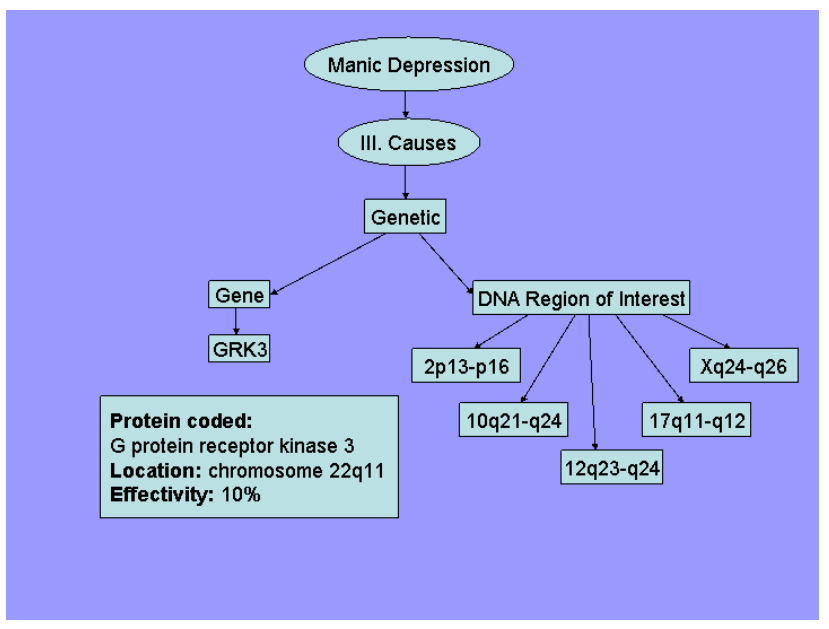

Figure 5: Genetical causes of manic-depression, current research

If a new gene is found on one of the already identified DNA regions of interest, our model will now have four instead of five instances of the term 'DNA Region of Interest' and one more instance of the term 'gene' (see
Figure 6). Because of the length of DNA sequence it obviously is much easier for a researcher to target a specific area of a chromosome such as 2p13-16 than the whole chromosome 2. Further research, may allow her/him to narrow down the region of interest to, for example 2p14-15. Because of the agreed semantics in a shared ontology it will be easier for the next person to continue the research in the same direction and possibly to locate the gene of interest.

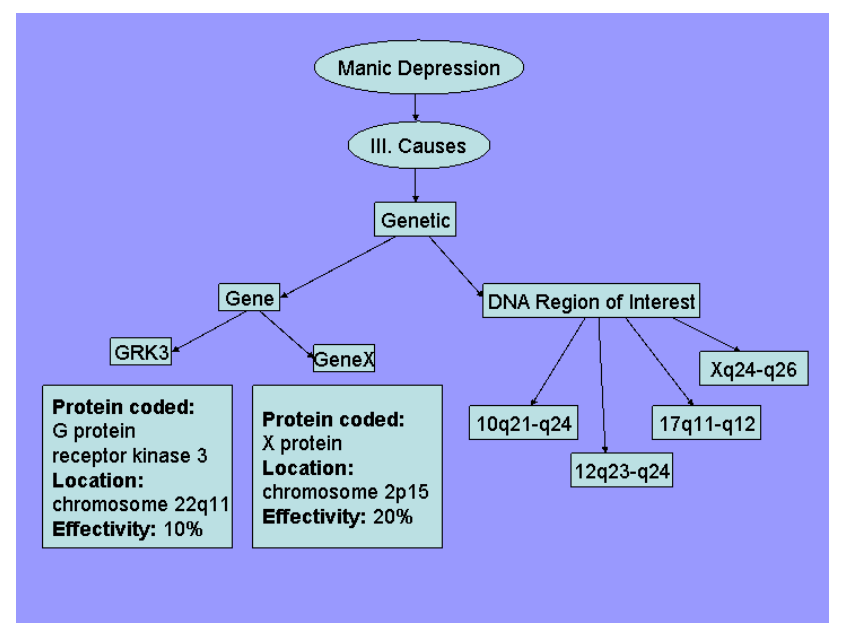

Figure 6: Genetic causes of manic-depression, future research if gene of interest found on chromosome 2 (position p15 and effective in $20 \%$ of manic depression cases)

This aspect of cooperation between different teams increases productivity by saving time and research resources.

\section{Conclusions and Further Work}

The development of an integrated ontology for the purpose of representing the active knowledge about human disorders has a number of obvious but quite important advantages:

- A computer-based ontology supports the work of scientists in gathering information on highly specific research topics of human disorders, and allows users on a world-wide basis to intelligently access new scientific information much more quickly;

- Shared knowledge improves research efficiency and effectiveness, as it helps (a) to avoid unnecessary redundancy in doing the same experiments, such as the examination of the same region of a DNA sequence, and (b) the determination of for example which part of DNA sequence needs to be further 
examined in order to find the gene responsible for a disease;

- Ontologies are the basis of interoperation, by allowing distributed but autonomous and heterogeneous resources to function in a world-wide cooperative environment: this makes it possible to split effectively a big task between different research teams;

- Constructing the data patterns combining different genetic and environmental causes and different disease types, will facilitate the sorting out of the exact combinations of the genetic and environmental factors involved as well as their individual influences on a specific complex disease type such as e.g. depression, thereby assisting medical professionals to diagnose, treat and possibly prevent the disorder.

Currently we are developing our ontology using DOGMA Modeler and trusted information resources such as OMIM and MayoClinic. Future work will enable our system to provide a user with a complete blueprint of a disease.

\section{Acknowledgments}

This paper has been finalized during Maja Hadzic's research visit at the VUB STARLab in Brussels. The authors would like to thank Prof. Robert Meersman, Sven Van Acker, Andriy Lisovoy and other research team members of VUB STARLab for helpful discussions and useful suggestions.

\section{References}

[1] Alejandro A. Schaeffer, Sandeep K. Gupta, K. Shriram, Robert W. Cottingham Jr. (1994): Avoiding Recomputation in Linkage Analysis. Human Heredity, vol. 44, pp. 225-237.

[2] Altman R., Bada M., Chai X.J., Whirl Carillo M., Chen R.O., Abernethy N.F. (1999): Riboweb: An Ontology Based System for Collaborative Molecular Biology. IEEE Intelligent Systems, vol. 14, no. 5, pp. 68-76.

[3] Andreas Baxevanis D. \& Francis Ouellette B. F. 2001, BIOINFORMATICS: A Practical Guide to the Analysis of Genes and Proteins, Wiley-Interscience.

[4] Barrett TB, Hauger RL, Kennedy JL, Sadovnick AD, Remick RA, Keck PE, McElroy SL, Alexander M, Shaw SH, Kelsoe JR (2003): Evidence that a single nucleotide polymorphism in the promoter of the $\mathrm{G}$ protein receptor kinase 3 gene is associated with bipolar disorder. Molecular Psychiatry, vol. 8, pp. 546-557.
[5] Bodenreider O. (2004): The Unified Medical language System (UMLS): integrating biomedical terminology. Nucleic Acids Res, vol. 32, no. 1, pp. 267-270.

[6] Craddock N, Jones I. (2001): Molecular genetics of bipolar disorder. The British Journal of Psychiatry, vol. 178, no. 41, pp. 128-133.

[7] Dyck Patricia, Chisholm Rex (2003): Disease Ontology, in: Sixth Annual Bio-ontologies Meeting Brisbane, 2003, pp.53-55.

[8] Gene Ontology Consortium (2002): Gene Ontology: tool for the unification of biology. Nat Genet, vol 25, pp. 25-29.

[9] Gruber, T. R. (1993): A Translation Approach to Portable Ontology Specifications. Knowledge Acquisition, vol. 5, no. 2, pp. 199-220.

[10] Halpin T. (2001): Information modeling and relational databases, $3^{\text {rd }}$ edition, Morgan-Kaufmann.

[11] Hattori E, Liu C, Badner JA, Bonner TI, Christian SL, Maheshwari M, Detera-Wadleigh SD, Gibbs RA, Gershon ES (2003): Polymorphisms at the G72/G30 gene locus, on 13q33, are associated with bipolar disorder in two independent pedigree series. American Journal of Human Genetics, vol. 72, no. 5, pp. 1131-1140.

[12] Liu J, Juo SH, Dewan A, Grunn A, Tong X, Brito M, Park N, Loth JE, Kanyas K, Lerer B, Endicott J, Penchaszadeh G, Knowles JA, Ott J, Gilliam TC, Baron M (2003): Evidence for a putative bipolar disorder locus on 2p13-16 and other potential loci on 4q31, 7q34, 8q13, 9q31, 10q21-24, 13q32, 14q21 and 17q11-12. Mol Psychiatry, vol. 8, no. 3, pp. 333-342.

[13] Meersman R. (1999): Semantic Ontology Tools in Information System Design. In: Ras Z., Zemankova M. (eds.): Proceedings of the ISMIS 99 Conference, Lecture Notes in Computer Science no. 1609, Springer Verlag, pp. 30-45.

[14] Montyne Frank (2001): The importance of formal ontologies: A case study in occupational health. In: d'Atri, A. \& Missikoff, M. (eds.): Proceedings of the OES-SEO 2001 Rome Workshop, Luiss Publications.

[15] Spyns P., Meersman R. \& Jarrar M. (2002): Data modelling versus Ontology engineering. SIGMOD Record, vol. 31, no. 4, pp. 7-12.

[16] Stevens R., Baker P., Bechhofer S., Ng G., Jacoby A., Paton N.W., Goble C.A., and Brass A. (2002): TAMBIS: Transparent Access to Multiple Bioinformatics Information Sources. Bioinformatics, vol. 16, no. 2, pp.184-186.

[17] Verheyen G., Raeymaekers P., Van Broeckhoven C. (1997): De genetica van stemmingsstoornissen, Cns Review, vol. 2, no. 2, pp. 7-12. 